\title{
Diagnóstico de la inserción de la ética en la carrera de medicina en Brasil
}

\author{
M.R.C.G. Novaes ${ }^{\text {a }}$ D. Guilhem ${ }^{\text {b }}$ L.C.G. Novaes ${ }^{\text {a }}$ F. Lolas ${ }^{c}$
}

Objetivo. Realizar el diagnóstico sobre la inserción de temas relacionados con la ética en cursos de pregrado de medicina en Brasil. Materiales y métodos. Estudio de corte transversal, retrospectivo histórico y exploratorio descriptivo. Se analizaron proyectos pedagógicos de 175 escuelas de Brasil. Se realizó una búsqueda activa en los documentos a través del análisis de aparición de términos con base en indexadores DeCS/MeSH. Los datos se correlacionaron con las informaciones relativas a los modelos pedagógicos de aprendizaje utilizados: metodologías activas (discusiones tutoriales en pequeños grupos y proyecto pedagógico integrado en módulos/ejes) o metodologías tradicionales (clases expositivas para grupo numeroso y proyecto pedagógico separado por disciplinas), y se realizaron los siguientes análisis: frecuencia de la inserción de temas agrupados y carga horaria media semestral de estos temas. Resultados. En los currículos analizados se observó que 95 cursos de pregrado en medicina utilizaban metodologías tradicionales de aprendizaje. Los temas agrupados más frecuentes fueron: ética médica; conducta profesional; deontología médica; procedimientos éticos; teología, filosofía, sociología y humanismo; ética en investigación; bioética en salud colectiva; y donación, muerte y eutanasia. La prueba de correlación de Spearman entre la presencia de metodologías activas y tradicionales fue $P_{S}$ $=0,814(\alpha=0,014 ;$ IC 95\%). Conclusión. Más del 80\% de escuelas con metodología activa presentan una carga horaria sobre ética de 50-100 h, mientras que más del 60\% de escuelas con metodología tradicional destinan una carga horaria inferior a $50 \mathrm{~h}$ para estos temas.

Palabras clave. Bioética. Brasil. Educación médica. Ética. Humanidades. Práctica profesional.

\section{Diagnosis of ethics issues in undergraduate medicine curriculum in Brazil}

Aim. To overview of ethics issues inclusion in CGM in Brazil, both in courses with active learning processes and traditional lecture learning methodologies. Materials and methods. This is a cohort, retrospective historical and descriptive exploratory study. We analyzed curricula and teaching projects of 175 medical schools of Brazil. The remaining 31 CGM were excluded either because of incomplete information or refusal to participate in the study. Active search for information was done on institutional sites and on documents through institutional occurrence analysis of terms based on DeCS/MeSH descriptors. Information was correlated according to learning educational model: active learning methodologies (MA) -tutorials discussions with integrated curriculum into core modules- or traditional lecture learning methodologies (MT) - with classes for large groups separated by disciplines-and reviewed by: OCcurrence frequency of ethical themes and average hourly load per semester of these themes. Results. It was observed that 95 CGM used MT. The most frequent ethical themes were: medical ethics; professional conduct; medical responsibility; ethical procedures; theology, philosophy, sociology and humanism; ethics in research; bioethics in public health; and organ transplantation, death and euthanasia. Spearman's rank correlation test between MA and MT occurrence resulted $P_{S}=0814$ $(\alpha=0.014 ; 95 \%$ Cl). Conclusion. Over $80 \%$ of MA schools utilizing thematic working hourly load between 50-100 h while more than $60 \%$ of MT schools used less than $50 \mathrm{~h}$ in curriculum time.

Key words. Bioethics. Brazil. Curriculum. Education. Ethics. Humanities. Medicine. Professionalism. a Curso de Medicina. Escuela Superior en Ciencias de la Salud. ESCS/FEPECS/GDF.

b Departamento de Enfermería. Universidad de Brasilia. Brasilia, Brasil. Departamento de Bioética. Universidad de Chile. Santiago, Chile.

\section{Correspondencia}

Prof. Maria Rita C.G. Novaes. Comitê de Ética em

Pesquisa. FEPECS/SES-DF. SHIS-QI-09, conj. 06, casa 14. Lago Sul, Brasília, Brazil. CEP: 71.625.060.

E-mail

ritanovaes@ig.com.br 


\section{Introducción}

La enseñanza universitaria en Brasil tuvo inicio con la llegada de la monarquía en el año 1808, cuando João VI, por medio de cartas regias, autorizó la creación de las dos primeras facultades de medicina en las ciudades de Salvador y Río de Janeiro [1]. No existen documentos de la época que mencionen la inclusión de temas relacionados con la ética, humanidades y profesionalismo en los currículos adoptados en aquel entonces.

Actualmente existen 175 facultades de medicina en Brasil, país considerado el segundo del mundo en número de escuelas médicas, tras la India, con 202 [2-4]. El tema de la ética se introdujo en el currículo médico de los cursos brasileños a finalales del siglo xix como parte de una disciplina denominada 'medicina legal'. La bioética, por ser una disciplina contemporánea, se introdujo recientemente en los currículos, durante los últimos diez años [5-8].

Diferentes entidades han considerado la necesidad de llevar a cabo un cambio efectivo en el proceso educativo de los estudiantes de medicina. La Conferencia Mundial de Educación Médica, realizada en Edimburgo (Escocia) en 1988, divulgó la 'Declaración de Edimburgo' por medio de la cual se recomendó que el desarrollo de la competencia profesional debería asociarse al reconocimiento de valores sociales, culturales y éticos, además de promover la interacción entre las escuelas médicas y los servicios básicos y comunitarios de salud [5-9].

En Brasil, puede mencionarse el papel destacado de la Asociación Brasileña de Educación Médica (ABEM) en la reformulación de la educación médica [4]. Se desarrollaron también iniciativas en este sentido por parte de la Organización Mundial de la Salud (OMS). Los principales documentos internacionales -entre los cuales pueden mencionarse el Learning Objectives for Medical Student Education de la Association of American Medical Colleges, divulgado en 1998, en Estados Unidos; el Tomorrow's doctors, elaborado por el General Medical Council, revisado el 2003, en Reino Unido, así como diversas guías de la Association for Medical Education in Europe (AMEE)- también recomendaron la adopción de objetivos claros y legitimaron las bases para una orientación curricular que busque el aprendizaje de conocimientos y el desarrollo de habilidades éticas y humanistas en el contexto profesional $[8,9]$.
Las Directrices Curriculares para Cursos de Pregrado en Medicina en Brasil fueran homologadas por el Consejo Federal de Medicina en el año 2001, y legitimaron las bases para la actual formación médica orientada hacia la adquisición de conocimientos generales, éticos y humanistas en la perspectiva de la integralidad de la asistencia, con responsabilidad social y compromiso con la ciudadanía [4]. En Brasil, el primer curso de la Facultad de Medicina es el bachillerato o preuniversitario, como en España.

En el 2002, el Ministerio de Salud y el Ministerio de Educación instituyeron el Programa Nacional de Incentivo a Cambios Curriculares en las Facultades de Medicina (PROMED). La propuesta tiene como objetivo incentivar a las facultades de medicina de todo el país para que incorporen cambios pedagógicos significativos en sus currículos [10-12].

Las innovaciones metodológicas y curriculares apuntan a la necesidad imperativa de insertar la temática relacionada con la ética, sugiriendo cambios en el proceso de aprendizaje. Estos aspectos son fundamentales para mantener el compromiso de la escuela con la asistencia a la salud y la valorización del desarrollo de habilidades y actitudes éticas. Además de esto, buscan ofrecer oportunidades para que los estudiantes puedan conocer la realidad del entorno social y del imaginario colectivo que circundan la percepción sobre las enfermedades, con el objetivo de formar profesionales con sensibilidad para analizar el contexto en el cual va a desarrollar sus actividades [4].

$\mathrm{El}$ afrontamiento de los problemas que involucran aspectos morales conflictivos en situaciones reales de la asistencia a la salud demuestra la insuficiencia de basar las decisiones en el código de ética profesional, documento que define la forma de actuar en términos de lo que se permite y lo que se prohíbe [5]. Es posible observar con frecuencia la inobservancia de las normas, aunque sean totalmente conocidas, así como las puniciones consecuentes a su infracción [6].

En este escenario, la bioética es una herramienta que contribuye a demostrar la necesidad de sobrepasar el límite deontológico y legal basado en normas y reglas, lo que permitirá efectuar una reflexión ética vinculada a la dimensión biopsicosocial del individuo [7]. De esta forma, la inclusión de los temas relacionados con la ética, bioética y humanización en una disciplina des- 
vinculada de la totalidad de informaciones requeridas para el proceso de toma de decisiones, descontextualizada y basada en el abordaje simplista del código de ética médica, estaría en desacuerdo con las directrices curriculares actuales.

La interacción entre los distintos y complejos conflictos que emergen de la práctica médica contemporánea ha llevado a la reformulación del papel de la ética en la medicina. La expansión del conocimiento médico, evidenciado por la marcada inclinación a la especialización, mejoró la calidad y expectativas de vida, pero con el coste del deterioro de la relación médico-paciente. El impacto de la ciencia y de la tecnología en la medicina colocó al alcance del médico alternativas de tratamiento que no estaban disponibles anteriormente, situación que ha creado nuevos conflictos, cuyas soluciones requieren respuestas también innovadoras $[8,9]$.

La deshumanización de la práctica médica debe analizarse desde distintas vertientes, entre ellas el punto de vista ético que considera la relación médico-paciente, así como desde la epistemología. Pertenece a las escuelas médicas la responsabilidad de proponer propuestas capaces de solucionar los problemas relacionados con la formación del futuro profesional médico en lo que se refiere a la formación de la conciencia ética. El equipo de salud asume la responsabilidad de cuidar de la salud de individuos y poblaciones humanas, lo que hace imprescindible la adopción de una postura crítica que permita reevaluar constantemente sus acciones. Esto permitirá cuestionarlas y direccionar la práctica cotidiana y el proceso de toma de decisiones en base a principios humanistas, holísticos y antropológicos $[9,10]$.

Por tanto, el médico que la sociedad necesita es un profesional con sólida formación general, capacitación técnica en cuidados básicos, entrenamiento para actuar en los diferentes niveles de atención a la salud, y elevada formación ética y humanista, aspectos que lo volverán apto para ejercer su profesión con responsabilidad social y capacidad técnica [11].

La sedimentación de la postura ética y del pensamiento crítico de los estudiantes debería iniciarse en las escuelas médicas por medio del desarrollo de actividades capaces de promover la discusión incluso en el ciclo académico-profesional. Todas las disciplinas, módulos o actividades del currículo médico deberían integrarse con las áreas de humanidades, representadas por los contenidos relacionados con filosofía, ética, psicología, antropología, artes, sociología e historia, con el fin de prevenir el riesgo de un reduccionismo humanístico y ético en la práctica clínica contemporánea $[11,12]$.

Esta investigación tuvo como objetivo realizar un diagnóstico de la inserción de la ética en el contexto de las humanidades y el profesionalismo en los cursos brasileños de pregrado en medicina, relacionándolo con los dos principales modelos de enseñanza-aprendizaje adoptados por las escuelas médicas: el método tradicional (MT) y el método activo (MA).

\section{Materiales y métodos}

\section{Delineamiento del estudio}

Estudio de corte transversal, retrospectivo histórico y exploratorio descriptivo. El estudio se realizó entre febrero de 2007 y febrero de 2008. La casuística estaba formada por 175 escuelas médicas inscritas en el Instituto Nacional de Estudios e Investigaciones Educacionales, vinculado al Ministerio de Educación, que constituyó el universo de análisis.

La investigación se realizó en dos etapas. Primero se efectuó el análisis cualitativo del modelo pedagógico predominante en el curso y el análisis cuantitativo relacionado con la inclusión de la temática, cuya variable de estudio fue la presencia de términos indexadores en el texto de los proyectos pedagógicos y de los currículos médicos. Los 127 términos utilizados para esta etapa de la investigación se describen en la tabla. El criterio de selección de los términos se definió utilizando la terminología de dos de los índices existentes en el área de ciencias de la salud: Descriptores en Ciencias de la Salud (DeCS) y Medical Subject Heading (MeSH). Para la búsqueda de los indexadores se consideró el radical del término; por ejemplo, 'abort', que puede corresponder a las palabras 'aborto', 'abortado', 'abortivo', 'abortista...

Basándose en los temas y subtemas elegidos, se agruparon los 127 indexadores en ocho grupos principales para los cuales los temas convergían (temas y subtemas agrupados): ética médica; conductas clínica y profesional; deontología médica y relación médico-paciente; procedimientos 
éticos y avances tecnológicos; teología, filosofía, sociología y humanismo; ética en investigación; bioética en salud colectiva; y donación, muerte, eutanasia y distanasia.

Los términos -considerados las variables del estudio- se colocaron en tablas para cada una de las escuelas médicas y se computaron de acuerdo con su aparición por unidad educacional, por disciplina en los cursos con MT, o por módulo $\mathrm{u}$ objetivo en los cursos con MA.

El acceso a los proyectos pedagógicos y currículos de las facultades de medicina se llevó a cabo por medio de los sitios institucionales de las facultades, del Ministerio de Educación y del Consejo Federal de Medicina. Los documentos impresos se transformaron en versión electrónica para la realización de las búsquedas efectuadas.

De las 175 escuelas médicas existentes en Brasil, 144 se incluyeron en la muestra de la investigación. Se excluyeron las 31 restantes porque no poseían todas las informaciones necesarias o por no haber aceptado participar en el estudio.

Se analizaron los currículos de las escuelas que compusieron la muestra y los términos se anotaron en base al valor cuantitativo de aparición, de forma decreciente. A través del criterio de los cuartiles centrales p50 y p75 se establecieron las escuelas que serían incluidas en la segunda fase de la investigación y sometidas al análisis cualitativo. Se excluyeron las que presentaban datos inconsistentes o conflictivos y las que estaban en los outliers y fuera del intervalo de confianza $(p<0,05)$.

Para la segunda fase de la investigación, los datos de las 54 escuelas incluidas se complementaron con informaciones obtenidas por correo electrónico, por medio del cual se envió el cuestionario a los coordinadores de las facultades. Las escuelas cuyos formatos se presentaban inconclusos fueron contactadas por teléfono, a través de sus directores.

El cuestionario se elaboró con preguntas abiertas y cerradas, con un tiempo medio para cumplimentarlo de unos 15 minutos. El objetivo de este instrumento era evaluar las siguientes variables:

- Obligatoriedad de la inserción de temas relacionados con la ética en las distintas unidades curriculares.

- Frecuencia del tema por módulo o disciplina.

- Carga horaria media utilizada para desarrollar la temática en cada semestre por unidad curricular.
- Metodología utilizada para la discusión de los temas.

- Temas de bioética y ética más frecuentemente discutidos en las actividades.

- Modelo pedagógico adoptado por el curso analizado.

El cuestionario aplicado se validó por medio del análisis de tres profesores de la Escuela Superior de Ciencias de la Salud (ESCS), en Brasilia, Brasil.

Las respuestas de los 54 coordinadores de los cursos respecto a la metodología utilizada (activa frente a tradicional) se correlacionaron con las observaciones documentales para la validación interna de la investigación.

Los análisis se realizaron utilizando el programa SPSS v. 15.1, por medio de estadísticas descriptivas y analíticas. Los resultados se presentaron en forma de tablas y gráficos. Las significaciones se contrastaron a través de la realización de pruebas de hipótesis de probabilidad de significación (valor $p$ ). El intervalo de confianza adoptado fue $\alpha=0,05$.

\section{Aspectos éticos de la investigación}

El proyecto fue evaluado y aprobado por el Comité de Ética en Investigación de la FEPECS/ SES-DF, bajo el protocolo 052/07. Los coordinadores de las facultades de medicina, o profesional por ellos indicado para cumplimentar el cuestionario, participaron voluntariamente en la investigación y firmaron el formato de consentimiento libre y esclarecido, que remitieron por correo electrónico o fax. Se aseguró la confidencialidad de las instituciones participantes y de las informaciones obtenidas.

\section{Resultados}

En lo que se refiere al método de enseñanzaaprendizaje utilizado por los cursos de medicina, se observó que el 65,97\% $(n=95)$ de las escuelas adoptaban el modelo pedagógico tradicional (MT), y el 34,03\% $(n=49)$, metodologías activas (MA) de aprendizaje.

Para evaluar la significación estadística de la incidencia por porcentaje del tipo de metodología utilizada -MA o MT- se recurrió a la prueba binominal, considerando $\alpha=0,05$. La razón exis- 


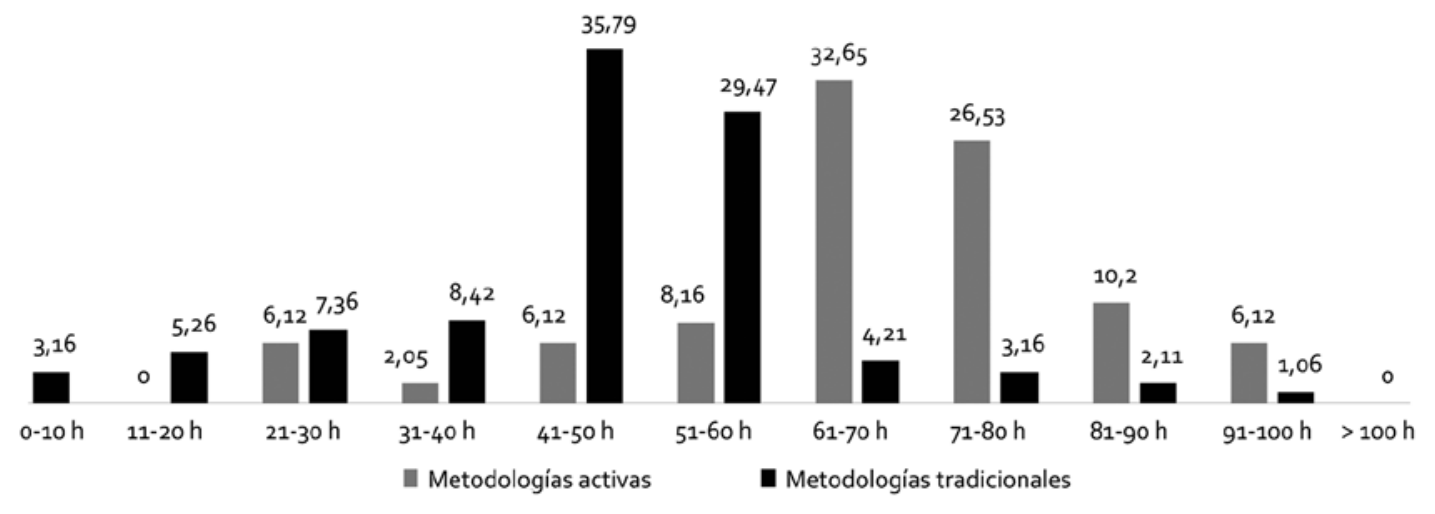

Figura. Distribución porcentual de la carga horaria destinada a las temáticas relacionadas con la ética y la bioética.

tente entre la proporción del número de cursos MT/MA fue de $r=1,94$. El análisis estadístico inferencial indica que el porcentaje de escuelas que adoptaron MA es significativamente diferente del $50 \%(p=0,043 ; n=144)$. La comparación entre la metodología declarada en los cuestionarios dirigidos a los coordinadores de los cursos $(n=$ 54) y aquella verificada por el análisis cualitativo se realizó mediante la prueba de correlación $V$ de Cramer $(V=0,89 ; \alpha=0,01 ; p=0,03)$.

Respecto a la inserción curricular de los temas de ética en el contexto de humanidades y profesionalismo, el resultado de la búsqueda activa de términos indexadores en los 144 cursos médicos analizados se presenta en la tabla. La prueba de correlación de Spearman entre las ocurrencias MA y MT resultó de $P_{S}=0,814$ ( $\alpha=0,014 ;$ IC 95\%).

La distribución de la carga horaria de los cursos de pregrado en medicina por metodología para las temáticas de ética en el contexto de humanidades y profesionalismo se muestra en la figura. Más del $80 \%$ de las escuelas que utilizan MA presentan una carga horaria de la temática de $50-100 \mathrm{~h}$, mientras que más del $60 \%$ de las escuelas con MT destinan una carga horaria para la temática inferior a $50 \mathrm{~h}$.

\section{Discusión}

La introducción de las temáticas relacionadas con la ética en los cursos de pregrado en medicina busca formar médicos capaces de actuar respetando los derechos de los pacientes y observando los aspectos relacionados con las dimensiones biopsicosociales, y no sólo capaces de curar la enfermedad. Además, la temática contribuye al desarrollo de habilidades y actitudes que capacitarán a los profesionales a afrontar los distintos conflictos que emergen de la práctica cotidiana de la medicina.

No existe consenso sobre cuál sería el tipo de metodología más apropiado para la enseñanza de los contenidos en esta área. Algunos autores apuntan que el abordaje debería plantearse mediante métodos activos de aprendizaje, con especial énfasis en la discusión de los conflictos bioéticos, que deberían contextualizarse en los diferentes campos de la ética social, deontológi$\mathrm{ca}$, de la salud y de los avances científicos que repercuten en la vida en sociedad [12-15]. También postulan que esta discusión debería ocurrir durante todo el curso, empezándose en el primero semestre. Los diferentes conflictos que engloban cuestiones éticas, morales y actitudinales, sea por parte del estudiante, del equipe de salud, del paciente, de la familia o de la comunidad/sociedad, deberían presentarse y discutirse como estrategia para aproximar la realidad en la cual se desarrollarán las actividades profesionales [12-15].

La reflexión ética debería integrar tanto el ciclo básico como el profesional, cuando el proyecto pedagógico posibilita la integración curricular. Existe además la defensa de que el contenido necesita relacionarse con la práctica y, por tanto, estos temas se asimilarían mejor si se abordaran predominantemente durante el ciclo profesional 
Tabla. Frecuencia de los indexadores de temas y subtemas agrupados (TSA) relacionados con la ética, incluidos en proyectos pedagógicos y currículos médicos brasileños: metodología tradicional (MT; $n=95$ ) y metodología activa (MA; $n=49$ ).

\begin{tabular}{|c|c|c|c|c|}
\hline Términos indexadores & $\begin{array}{l}\text { Temas } \\
\text { (TSA) }\end{array}$ & $\begin{array}{c}\text { Curso } \\
\text { MA }\end{array}$ & $\begin{array}{c}\text { Curso } \\
\text { MT }\end{array}$ & Total \\
\hline $\begin{array}{l}\text { Certificación, código (de ética médica), fiabilidad, confianza, } \\
\text { dignidad, dilemas, error (médico), falsedad, fidelidad, juicio, } \\
\text { justicia, legal (legalidad), legislación, ley, moral, norma (ley), } \\
\text { pericia, permiso, principio (ético), publicidad, racionalidad, } \\
\text { veracidad, verdad }\end{array}$ & Ética médica & 152 & 179 & 331 \\
\hline $\begin{array}{l}\text { Aceptación, actitud, certeza, compasión, comunicación, } \\
\text { conducta (actitud), concienciación, escucha, gratitud, lucro, } \\
\text { privacidad, postura, reflexión, remuneración, reparación (daño), } \\
\text { responsabilidad, secreto, sensibilidad, sigilo (profesional), } \\
\text { solidaridad, ventaja, vínculo, virtud }\end{array}$ & $\begin{array}{l}\text { Conductas clínica } \\
\text { y profesional }\end{array}$ & 45 & 37 & 82 \\
\hline $\begin{array}{l}\text { Acto (médico), deontología, deber, derecho (médico), telepatía, } \\
\text { error (médico), negligencia, calidad (de vida), relación (médico- } \\
\text { paciente), respeto }\end{array}$ & $\begin{array}{l}\text { Deontología médica y } \\
\text { relación médico-paciente }\end{array}$ & 38 & 22 & 60 \\
\hline $\begin{array}{l}\text { Avances, autonomía, beneficencia, beneficio, bioseguridad, } \\
\text { imprudencia, (no) maleficencia, procedimiento (ético), riesgo } \\
\text { (frente a beneficio), tecnológico }\end{array}$ & $\begin{array}{l}\text { Procedimiento ético y } \\
\text { avances tecnológicos }\end{array}$ & 32 & 14 & 46 \\
\hline $\begin{array}{l}\text { Antropología, creencia, cultura, desigualdad, diferencia, Dios, } \\
\text { dogma, fe, filosófico, histórico, holístico, humanismo (humano, } \\
\text { humanidad, humanista), imaginario, opresión, perdón, religión, } \\
\text { sacralidad (de la vida), santo, social, sociología, teología, valores } \\
\text { (culturales, religiosos, sociales y éticos) }\end{array}$ & $\begin{array}{l}\text { Teología, filosofía, } \\
\text { sociología y humanismo }\end{array}$ & 19 & 10 & 29 \\
\hline $\begin{array}{l}\text { Cobayos (animales y humanos), código (Nuremberg), comité } \\
\text { (de ética en investigación), conflicto, confidencialidad, } \\
\text { consentimiento, declaración (de Helsinki), esclarecimiento, } \\
\text { interés, investigación (en humanos), publicidad, resolución } \\
\text { (CNS 196/96), secreto }\end{array}$ & Ética en investigación & 17 & 9 & 26 \\
\hline $\begin{array}{l}\text { Aborto, distanasia, donación, eutanasia, muerte, trasplante, } \\
\text { vida, vivir }\end{array}$ & Bioética en salud colectiva & 19 & 7 & 26 \\
\hline $\begin{array}{l}\text { Acceso (a los servicios), bioética, ciudadanía, colectiva (salud), } \\
\text { derecho (a la asistencia a la salud), discriminación, equidad, } \\
\text { igualdad, minoría, sexualidad (ético), universalidad }\end{array}$ & $\begin{array}{l}\text { Donación, muerte, } \\
\text { eutanasia y distanasia }\end{array}$ & 13 & 11 & 24 \\
\hline
\end{tabular}

que todavía sigue existiendo en currículos tradicionales $[6,8,11]$.

En el año 2001, 103 facultades de medicina brasileñas habían introducido las temáticas relacionadas con la ética en los currículos. Las metodologías de enseñanza más utilizadas eran las consideradas tradicionales e incluían clases expositivas, discusión de casos y presentación de seminarios, entre otras modalidades [10-12]. Comparando este escenario con los datos divulgados en el año 1992, es posible afirmar que hubo una expansión significativa de la enseñanza de la ética y la bioética en los cursos, aunque el análisis de la carga horaria en estos períodos demuestra apenas un pequeño aumento de horas destinadas a estos temas. Aun así, existe actualmente una tendencia prometedora de valorización de contenidos de la ética, cuyos resultados futuros serán, indudablemente, positivos [10-12].

En este estudio fue posible observar que las facultades de medicina brasileñas discuten actualmente temas relacionados con los aspectos 
de la ética en investigación, conductas clínicas y profesionales, implicaciones filosóficas y deontológicas de la relación médico-paciente, conflictos relacionados con la muerte, eutanasia y distanasia, procedimientos y avances tecnológicos en el área de genética, nuevos fármacos y procedimientos; bioética y salud colectiva, y otras temas relacionadas como teología, sociología de la desigualdad y opresión, filosofía, antropología y humanismo. Estos temas se tratan en diferentes períodos de formación en las facultades de medicina investigadas.

Además, existen frecuencias distintas sobre la inclusión de temas relacionadas con la ética y su distribución en los diferentes períodos del curso, cuando se analizan los currículos de dos cursos. Persiste la pregunta: ¿la inclusión de temas variados y una mayor carga horaria en los currículos contribuirían a formar médicos capaces de desarrollar actitudes y prácticas mas respetuosas y éticas? Todavía no es posible comprobar que estos temas modifican realmente las posturas adoptadas por los futuros profesionales, pero las escuelas médicas deben incluir reflexiones que posibiliten la formación ética y humanista de los profesionales y, de esta forma, contribuir a mejorar la calidad de la asistencia médica brindada a la sociedad brasileña.

En conclusión, la evaluación de la inserción de la ética en las facultades de medicina en Brasil favoreció la obtención de informaciones que pueden contribuir a la discusión y revisión del currículo actualmente utilizado. La reflexión ética puede ser beneficiosa para la modificación del panorama de atención médica en el contexto de la salud colectiva.

Ante los resultados encontrados, se recomienda profundizar y sistematizar la discusión relacionada con estos temas en los cursos de graduación en medicina. Paralelamente, resulta imprescindible la reflexión sobre las metodologías y recursos didácticos utilizados como forma de sedimentar los conocimientos y temas discutidos. Algunas de las posibles alternativas serían: discusión de películas que aborden temas relacionados con los contenidos, análisis de materias periodísticas - medios escritos y televisión-, análisis de imágenes -cuadros, fotos, etc.y reflexión sobre narrativas históricas y literarias [16]. A partir de las situaciones presentadas será posible reflexionar y correlacionar los temas y conceptos de bioética en general, además de la ética en investigación y ética profesional discutida en las actividades prácticas del plan curricular de la carrera de medicina, para que haya un crecimiento y fortalecimiento de la reflexión ética de los estudiantes.

Una mirada ética y humanística permitirá a los médicos una mejor comprensión del pasado, un mejor análisis del presente y una mejor visión del futuro, lo que permitirá lograr una perspectiva independiente de la tecnología, asociando el juicio humano a las decisiones técnicas.

\section{Bibliografía}

1. Tonelli E. Escolas médicas. Jornal da Associação Médica de Minas Gerais 2005: 7.

2. Povoa L, Andrade MV. Distribuição geográfica dos médicos no Brasil; uma análise a partir de um modelo de escolha locacional. Cad Saude Pública 2006; 8: 1555-64.

3. Brasil. Ministério da Educação. Diretrizes curriculares nacionais para os cursos de graduação em Medicina; 2001.

4. Associação Brasileira de Educação Médica (ABEM). URL: http://www.abem-educmed.org.br.

5. Feuerwerker LCM. Mudanças na educação médica e residência médica no Brasil. 1 ed. São Paulo: Hucitec/Rede Unida; 1998.

6. Mclean M. The small group in problem-based learning: more than a cognitive 'learning' experience for first-year medical students in a diverse population. Med Teach 2006. URL: http://taylorandfrancis.metapress.com/ media/c28459nnth2qxka1tdtx/contributions/r/6/6/1/ r6610v7p428x47rw.pdf. [20.12.2006].

7. Goldie J, Schwartz L, McConnachie A, Morrison J. The impact of three years' ethics teaching, in an integrated medical curriculum, on students' proposed behaviour on meeting ethical dilemmas. Med Educ 2002; 36: 489-97.

8. General Medical Council (GMC). Tomorrow's doctors -regulating doctors ensuring good medical practice. URL: http://www.gmc-Uk.org/education/undergraduate/undergraduate_policy/tomorrows_doctors.asp. [12.10.2007].

9. Association of American Medical College (AAMC). Learning objectives for medical student education: guidelines for medical schools. URL: https://services. aamc.org/Publications/index.cfm?fuseaction=Product. displayForm\&prd_id=198\&prv_id=239. [12.10.2007].

10. Chedwidden WR. A problem-based learning pathway 
for medical students: improving the process through action research. Ann Acad Med 2006; 35: 642-6.

11. Muñoz D, Muñoz DR. O ensino da ética nas faculdades de medicina do Brasil. Revista Brasileira de Educação Médica 2003; 27: 114-24.

12. Gallian DMC. A (re)humanização da medicina. URL: http://www.priory.com/psych/galli0500.htm. [05.03.2007].

13. Miles SH, Lane LW, Bickel J, Walker RM, Cassel CK. Medical ethics education: coming of age. Acad Med 1989; 64: 705-9.
14. Self DJ, Wolinsky FD, Baldwin DC. The effect of teaching medical ethics on medical students' moral reasoning. Acad Med 1989; 64: 755-9.

15. Hebert PC, Meslin EM, Dunn EV. Measuring the ethical sensitivity of medical students: a study at the University of Toronto. J Med Ethics 1992; 18: 142-7.

16. Guilhem D, Diniz D, Zicker F. Pelas lentes do cinema: bioética e ética em pesquisa. Brasília: Letras Livres/Editora UnB; 2007. 